(2) Open Access Full Text Article

REVIEW

\title{
Review of hydroxypropyl cellulose ophthalmic inserts for treatment of dry eye
}

This article was published in the following Dove Press journal:

Clinical Ophthalmology

10 May 20II

Number of times this article has been viewed

\section{Theresa Nguyen \\ Robert Latkany}

Dry Center, Physician Eyecare of New York, New York, NY, USA
Correspondence: Robert Latkany

150 East 32nd Street Suite 102,

New York, NY 10016, USA

Tel + I 2126892020

Fax + I 2126892954

Email relief@dryeyedoctor.com
Abstract: Dry eye syndrome is a prevalent disease that affects visual acuity, activities of daily living, and quality of life. A number of contributory factors affect the severity of dry eye syndrome, including autoimmune disease, environmental surroundings, contact lens use, hormonal changes, anatomical features, chronic inflammation, infections, and iatrogenic factors, such as medications or surgery. Symptoms may include intermittent or constant blurry vision, discomfort, burning, foreign body sensation, hyperemia, dryness, and photophobia. The severity of dry eye syndrome can range from very mild disease to extremely severe cases with vision-threatening consequences. A variety of dry eye treatment modalities exist to address the different causes, symptoms, and consequences of ocular surface disease, including artificial tears, lubricating gels, ophthalmic inserts, anti-inflammatory drops, and surgical procedures. In this paper, an assortment of literature pertaining to the treatment of dry eye syndrome, in particular hydroxypropyl cellulose ophthalmic inserts, is reviewed. These inserts can be used effectively as monotherapy, or in conjunction with other therapies, and should be considered in the treatment of dry eye syndrome.

Keywords: hydroxypropyl cellulose ophthalmic inserts, dry eye, punctal occlusion, ocular surface disease

\section{Introduction}

Dry eye syndrome is a multifactorial disease of the tears and ocular surface that results in symptoms of discomfort, burning, itching, foreign body sensation, dryness, pain, photophobia, hyperemia, visual disturbance, and tear film instability, with potential damage to the ocular surface. ${ }^{1}$ A number of contributory factors affect the degree of dry eye syndrome, including autoimmune disease, environmental surroundings, contact lens use, hormonal changes, vitamin deficiencies, anatomical features, corneal dystrophies, chronic inflammation, infections, and iatrogenic factors, such as medications, radiation, and surgery. ${ }^{1,2}$ Dry eye can be classified into five types, ie, aqueous-deficient, mucodeficient, lipodeficient, epitheliopathic, and eyelid-eye incongruent. ${ }^{3}$ The International Dry Eye Workshop has classified dry eye etiology as either aqueous-deficient or evaporative. ${ }^{1}$ Aqueous deficiency is further classified as Sjogren-related or non-Sjogren-related dry eye, and evaporative is divided into intrinsic causes, including meibomian oil deficiency or lid disorders, and extrinsic causes, such as vitamin A deficiency, contact lens wear, or topical preservatives. Diagnosis of dry eye syndrome include subjective symptoms, as well as clinical signs, including punctate epithelial staining, tear film appearance and volume, tear film breakup time, tear film normalization test, and Schirmer tear test. ${ }^{1-3}$ It has been found that dry eye 
patients have reduced tear meniscus dynamics and smaller meniscus volume. ${ }^{4}$ Symptoms can be further classified by using one of a number of questionnaires, including the Ocular Surface Disease Index, a validated standardized instrument that measures disease severity and patient quality of life on a numerical scale in patients with dry eye syndrome. The severity of dry eye syndrome can range from very mild disease to extremely severe cases with vision-threatening consequences, such as ocular infections, perforations, endophthalmitis, and blindness. The severity of subjective symptoms does not necessarily correlate with the findings on clinical examination. ${ }^{5}$

Dry eye symptoms are one of the most common complaints seen in clinical ophthalmology settings. Populationbased surveys have indicated that dry eye disease affects millions of people worldwide. ${ }^{6}$ A multitude of dry eye treatment modalities exist to address the different causes, symptoms, and consequences of ocular surface disease, including artificial tears, lubricating gels, anti-inflammatory or immunosuppressant drops, steroids, autologous serum or platelet-rich plasma drops, and punctal plugs. Less common treatments include hormonal therapy, vitamin A, botulinum toxin, acupuncture, and antiviral agents. ${ }^{7}$ There are also surgical procedures to improve the quality of severe ocular surface disease, including repair of lid abnormalities, lid tarsorrhaphy, submandibular gland transplantation, and canalicular occlusion, which can include canalicular excision, punctal tarsorrhaphy, punctal patch, or punctal cautery, all of which preserve the natural tears, which to date are irreplaceable and remain better than artificial tears. ${ }^{8}$ Studies have shown that after punctal occlusion, patients have symptomatic improvement, as well as improvement in clinical signs of dry eye, tear film stability, and ocular surface retention. ${ }^{8-10}$

Hydroxypropyl cellulose ophthalmic inserts are another treatment available for moderate-to-severe dry eye syndrome. The chemical name for hydroxypropyl cellulose is cellulose, 2-hydroxypropyl ether. It is a tasteless, odorless, and physiologically inert substance, and is soluble in water below $38^{\circ} \mathrm{C}$. The ophthalmic inserts are sterile and translucent rods measuring $1.27 \mathrm{~mm}$ in diameter and $3.5 \mathrm{~mm}$ long. Each insert contains $5 \mathrm{mg}$ of hydroxypropyl cellulose, with no preservatives or any other ingredients. The medication is administered by placing a single insert into the inferior cul de sac of the eye beneath the base of the tarsus, not in opposition to the cornea, nor beneath the eyelid at the level of the tarsal plate. ${ }^{11}$ Hydroxypropyl cellulose ophthalmic inserts act by stabilizing and thickening the tear film and prolonging tear film breakup time, as well as lubricating and protecting the eye. The inserts are indicated especially for patients who continue to have dry eye symptoms after an adequate trial of therapy with artificial tears. ${ }^{11}$ They are also indicated for patients with keratoconjunctivitis sicca, exposure keratitis, decreased corneal sensitivity, and recurrent corneal erosions. ${ }^{11}$ The only contraindication to using this medication is hypersensitivity to hydroxypropyl cellulose. ${ }^{11}$

Several studies have been performed to evaluate the efficacy of hydroxypropyl cellulose ophthalmic inserts. In one study, 418 patients were evaluated with questionnaires after four weeks of treatment on hydroxypropyl cellulose ophthalmic inserts. ${ }^{12}$ The patient questionnaires were completed prior to initiation of treatment, and again after four weeks of treatment to assess the difference in severity of symptoms, ability to perform activities of daily living, quality of life, and frequency of discomfort in various environmental conditions. All participating patients were required to have a diagnosis of dry eye syndrome in both eyes, and a history of intermittent or regular artificial tear use. The patients were subdivided into patients without comorbid conditions and five groups of patients with comorbid conditions, including contact lens wearers, patients with cataracts, patients with glaucoma, patients who have undergone cataract surgery, and patients who have undergone prior laser-assisted in situ keratomileusis. Patients were excluded if they have clinically significant blepharitis, meibomian gland dysfunction, lid margin or ocular inflammation, ocular infection, laser keratorefractive surgery within 12 months, or any other ocular surgery within three months. Patients were also screened for hydroxypropyl cellulose hypersensitivity. The patients were prohibited from starting any new dry eye therapy that they were not previously using throughout the trial. The results of the study demonstrated that both patients with and without comorbidities had statistically significant improvements in symptoms of discomfort, burning, dryness, grittiness, stinging, and light sensitivity, as well as improvements in ability to perform activities of daily living and in quality of life. The three groups that showed the most improvement occurred in contact lens wearers, patients with prior cataract surgery, and patients with prior laser-assisted in situ keratomileusis. Although patients with only glaucoma or cataracts did not show as much improvement as the three previously mentioned groups, they still showed a statistically significant improvement.

A prospective study was published in which enrolled patients diagnosed with bilateral dry eye and a history 
of artificial tear use completed questionnaires at two visits, ie, at the beginning and at the end of a four-week registry. ${ }^{13}$ Most of the patients were women and over the age of 50 years, and age did correlate with a difference in response. There was more than $25 \%$ improvement in severity of discomfort, burning, dryness, grittiness, stinging, and sensitivity to light. Fifty-eight percent of the patients noted a statistically significant improvement in at least one symptom of the Ocular Surface Disease Index. Another study revealed similar findings, in which mean Ocular Surface Disease Index total scores improved by $21.3 \%{ }^{14}$ The study reported fairly good compliance, and the main adverse event that led to discontinuation was blurred vision.

A retrospective case series study was performed to determine whether patients experienced improvement in dry eye symptoms after use of hydroxypropyl cellulose ophthalmic inserts. ${ }^{15}$ Data from the study were obtained from medical records of patients who has been given a prescription for hydroxypropyl cellulose ophthalmic inserts within two years prior to the study. This included patients who were newly started on the inserts, as well as patients who were already being treated with the inserts prior to the two years. The patients were predominantly female and over the age of 60 years. Of the 80 participating patients, 73 were still currently using the insert, and most were using the inserts at least once a day (93.2\%). Some of the patients were using concomitant dry eye therapies, including topical antibiotics, artificial tears, and immunomodulators. The study reported a very low rate of adverse effects, which included blurred vision and foreign body sensation, and was only $2.5 \%$. The duration of therapy ranged from 10 days to over 26 years. The median duration of therapy with the insert was 5.3 years, and almost two-thirds of the patients continued to use the insert for more than two years. The long duration of use suggests that the medication is relatively safe, well tolerated, effective for long-term therapy, and successful in patients over the age of 60 years.

A more recent study evaluated whether subjective patientreported improvement in symptoms after treatment with hydroxypropyl cellulose ophthalmic inserts correlated with physician assessment of clinical signs. ${ }^{16}$ The study reported that over $75 \%$ of patients had an overall improvement in their symptoms after being treated for one month with hydroxypropyl cellulose ophthalmic inserts. It also reported that patient-reported improvements strongly correlated with and significantly predicted physician assessment of the condition, effectiveness of therapy, and willingness to use the inserts as adjunctive therapy.

A crossover study was performed in 22 patients with rheumatoid arthritis and moderate-to-severe keratoconjunctivits sicca, who were treated with slow-release artificial tear inserts. ${ }^{17}$ Half of the participants used one insert daily, and the other group used methylcellulose artificial tears four times a day. After two weeks, the participants were evaluated for subjective symptomatic changes and clinical staining. Thereafter, the participants all used artificial tears for one week. The groups were then switched to the opposite treatment and reassessed after a further two weeks. The study reported that $86 \%$ of the patients approved of the insert as therapy, and $64 \%$ of the patients preferred the inserts to the artificial tears. These results are similar to those of another study reporting that $75 \%$ of their patients with keratitis sicca preferred the slow-release artificial tear inserts as the main form of treatment. ${ }^{18}$ Despite having varying degrees of hand deformities and loss of dexterity because of rheumatoid arthritis, most patients reported no difficulty with insertion of the medication.

\section{Safety}

Although rheumatoid arthritis is not a contraindication to use of hydroxypropyl cellulose ophthalmic inserts, there are some drawbacks to the medication. Educating patients how to administer the inserts can be time-consuming and difficult. Possible side effects were reported in approximately $2.5 \%$ of patients, and these side effects included blurring of vision, foreign body sensation, ocular irritation or hyperemia, hypersensitivity, photophobia, eyelid edema, and caking or drying of viscous material on eyelashes. ${ }^{12,15}$ One study reported a corneal abrasion, but this was reportedly unrelated to ophthalmic insert usage. ${ }^{12}$ There are no reports of worsening of vision or dry eye symptoms, and most reported side effects have been mild and had no long-term or permanent adverse outcomes.

The most common reported side effect of hydroxypropyl cellulose ophthalmic inserts is blurred vision. The tear film is an important optical component that contributes to visual function. It has been reported that irregularities in the tear film can cause optical aberrations, fundus image degradation, decrease in contrast sensitivity, neural sharpness, and retinal vessel contrast. ${ }^{19,20}$ It has been observed that, as time increases after a blink, wavefront contours become more irregular and numerous, reflecting an increase in corneal wavefront aberrations, leading to perceptible degradation in vision. ${ }^{21,22}$ The 
effect of improving the tear film may be used as a diagnostic tool in detecting ocular surface disease in symptomatic and asymptomatic patients. ${ }^{23}$ While dry eye patients have blurred vision due to irregular corneal surface, the cause of blurred vision after insert application is most likely due to the viscous nature of the medication, not an insufficient tear film. In addition, although blurred vision was one of the most common side effects, most patients reported significant improvements in their ability to perform daily activities and visual tasks, particularly activities such as reading, watching television, working on the computer, and driving at night. ${ }^{12}$ This suggests that the blurred vision was not severe or visually impairing, and did not reduce quality of life. Blurred vision was also transient, and there were no reported cases of long-term decrease in vision.

\section{Conclusion}

In conclusion, there are various treatment modalities for dry eye syndrome available to eye care professionals, which can be used as monotherapy or in combination. There is evidence to suggest that with proper use and adequate patient education, hydroxypropyl cellulose ophthalmic inserts are an effective and safe treatment choice for dry eye syndrome. Most patients showed significant improvement in ocular symptoms and clinical signs, and many patients continued using hydroxypropyl cellulose ophthalmic inserts for several years alone or in conjunction with other dry eye therapies. There was no significant worsening in symptoms or any major long-term side effects of the medication. The inserts may be particularly helpful in patients who cannot tolerate preservatives or immunosuppressant drops, do not want to instill multiple artificial tears throughout the day, or still have an insufficient tear film despite other therapies. However, it is worth noting that several of the studies excluded patients with meibomian gland disease or blepharitis. It remains to be seen if the inserts help patients with evaporative aqueous tear loss due to meibomian gland dysfunction or blepharitis. One would think that both of these disease groups would benefit from using the inserts because there is often overlap of patients who also have dry eye syndrome. Nonetheless, hydroxypropyl cellulose ophthalmic inserts can be used effectively as monotherapy, or in conjunction with other therapies, and should be considered in the treatment of dry eye syndrome.

\section{Disclosure}

The authors have no proprietary or commercial interest in any of the materials discussed in this paper.

\section{References}

1. The definition and classification of dry eye disease: Report of the definition and classification subcommittee of the international dry eye workshop (2007). Ocul Surf. 2007;5:75-92.

2. Latkany R. Dry eyes: Etiology and management. Curr Opin Ophthalmol. 2008;19:287-291.

3. Latkany R, Lock B, Speaker M. Tear film normalization test: A new diagnostic test for dry eyes. Cornea. 2006;25:1153-1157.

4. Yuan Y, Wang J, Chen Q, Tao A, Shen M, Shousha M. Reduced tear meniscus dynamics in dry eye patients with aqueous tear deficiency. Am J Ophthalmol. 2010;149:932-938.

5. Nichols K, Nichols J, Mitchell G. The lack of association between signs and symptoms in patients with dry eye disease. Cornea. 2004;23: 762-770.

6. Schein OD, Munoz B, Tielsch JM, et al. Prevalence of dry eye among the elderly. Am J Ophthalmol. 1997;124:723-728.

7. Calonge M. The treatment of dry eye. Surv Ophthalmol. 2001; 45:S227-S239.

8. Murube J, Murube E. Treatment of dry eye by blocking the lacrimal canaliculi. Surv Ophthalmol. 1996;40:463-480.

9. Balaram M, Schaumberg D, Dana MR. Efficacy and tolerability outcomes after punctal occlusion with silicone plugs in dry eye syndrome. Am J Ophthalmol. 2001;131:30-36.

10. Yen M, Pflugfelder S, Feuer W. The effect of punctal occlusion on tear production, tear clearance, and ocular surface sensation in normal subjects. Am J Ophthalmol. 2001;131:314-323.

11. Lacrisert [Package insert]. Lawrenceville, NJ: Aton Pharmaceuticals Inc; 2007.

12. Luchs J, Nelinson D, Macy J. Efficacy of hydroxypropyl cellulose ophthalmic inserts (LACRISERT) in subsets of patients with dry eye syndrome: Findings from a patient registry. Cornea. 2010;29: $1417-1427$.

13. Koffler B, McDonald M, Nelinson D. Improved signs, symptoms, and quality of life associated with dry eye syndrome: Hydroxypropyl cellulose ophthalmic insert patient registry. Eye Contact Lens. 2010;36: 170-176.

14. McDonald M, D'Aversa G, Perry H, Wittpenn J, Donnenfeld E, Nelinson D. Hydroxypropyl cellulose ophthalmic inserts (Lacrisert) reduce the signs and symptoms of dry eye syndrome and improve patient quality of life. Trans Am Ophthalmol Soc. 2009;107:214-221.

15. Wander A, Koffler B. Extending the duration of tear film protection in dry eye syndrome: Review and retrospective case series study of the hydroxypropyl cellulose ophthalmic insert. Ocul Surf. 2009;7: 154-162.

16. Mcdonald M, D'Aversa G, Perry H, Wittpenn J, Nelinson D. Correlating patient-reported response to hydroxypropyl cellulose ophthalmic insert (LACRISERT) therapy with clinical outcomes: Tools for predicting response. Curr Eye Res. 2010;35:880-887.

17. Hill J. Slow-release artificial tear inserts in the treatment of dry eyes in patients with rheumatoid arthritis. Br J Ophthalmol. 1989;73: $151-154$.

18. Katz J, Kaufman H, Breslin C, Katz I. Slow release artificial tears and the treatment of keratitis sicca. Ophthalmology. 1981;88:78-81.

19. Tutt R, Bradley A, Begley C, Thibos L. Optical and visual impact of tear break-up in human eyes. Invest Ophthalmol Vis Sci. 2000;41: $4117-4123$.

20. Liu H, Thibos L, Begley C, Bradley A. Measurement of the time course of optical quality and visual deterioration during tear break-up. Invest Ophthalmol Vis Sci. 2010;51:3318-3326.

21. Montés-Micó R, Alió J, Muñoz G, Charman W. Temporal changes in optical quality of air-tear film interface at anterior cornea blink. Invest Ophthalmol Vis Sci. 2004;45:1752-1757.

22. Liu Z, Pflufelder S. Corneal surface regularity and the effect of artificial tears in aqueous tear deficiency. Ophthalmology. 1999;106:939-943.

23. Nilforoushan M, Latkany R, Speaker M. Effect of artificial tears on visual acuity. Am J Ophthalmol. 2005;140:830-835. 
Clinical Ophthalmology

\section{Publish your work in this journal}

Clinical Ophthalmology is an international, peer-reviewed journal covering all subspecialties within ophthalmology. Key topics include: Optometry; Visual science; Pharmacology and drug therapy in eye diseases; Basic Sciences; Primary and Secondary eye care; Patien Safety and Quality of Care Improvements. This journal is indexed on

PubMed Central and CAS, and is the official journal of The Society of Clinical Ophthalmology (SCO). The manuscript management system is completely online and includes a very quick and fair peer-review system, which is all easy to use. Visit http://www.dovepress.com/ testimonials.php to read real quotes from published authors. 